Computer Science

December 2016

\title{
How to Harness Endless Streams of Real-Time Data? Ryerson Researchers Explore "Immune Programming"
}

Did you really just buy $\$ 400$ sneakers with your credit card in Winnipeg - or are you in fact curled up at home in Toronto? Should that email from an online dating site be routed straight to your Inbox, or does it belong in your spam folder?

Fast internet and storage technologies allow us to collect and store massive amounts of online data. And we can then, as illustrated by the examples above, leverage it powerfully - whether that might involve detecting credit card fraud or spam filtering. To-date though, there's been a bit of a hitch. When programmers utilize traditional algorithms to classify data streams, they're hampered by a simple fact. Namely, it's difficult to individually identify every possible wrinkle in a scenario. For example, the possible signs of a fraudulent credit card transaction are myriad and highly individual too.

The key is of course intelligent systems - also known as machine learning models - which can gradually learn to make better predictions. At Ryerson, Jaspreet Bassan, who recently earned her MSc in Computer Science, devoted her graduate studies to exploring the merits of one particular model called immune programming.

What is it? Bassan explains that immune programming is an evolutionary machine learning technique that "takes inspiration from a biological immune system, to classify streaming data in real-time." In the credit card scenario, for example, she explains that via this model suspect transactions could be classified as "abnormal" and then aborted.

Bassan says her main contribution vis-à-vis the immune programming model was to verify its relevance for offline and real-time classification problems. "Since this algorithm is a supervised machine learning technique, that is, it uses labelled data to learn, my job was to develop a data labelling algorithm. I also designed the system's interface for real-time streaming data." Supervised by Ryerson's Dr. Marcus Santos, Bassan used Racket, a general-purpose programming language to program the system.

Last December, Bassan and Dr. Santos presented the Ryerson research at the 2016 IEEE Symposium Series on Computational Intelligence (SSCI 2016) in Athens, Greece. Their paper, titled, "Classifying Streaming Data using Grammar-based Immune Programming" is currently "In Press."

Ultimately, Bassan is keen on the functioning of the immune programming model, saying: "It inspires me to observe nature more carefully." She also stresses that potential applications stretch far beyond examples noted. "We could potentially use this research to analyze electricity demand or financial trends."

Asked about her experience at Ryerson, Bassan lists interacting with a diverse group of students as a teaching assistant among her personal highlights. She also credits a supportive faculty: "All this would have been impossible without my supervisor's constant guidance and I would like to take this opportunity to thank him big time." 